\title{
An integrated all-solid-state screen-printed potentiometric sensor based on a three-dimensional self-assembled graphene aerogel
}

\author{
Jinghui Li ${ }^{\mathrm{a}}$, Wei Qin ${ }^{\mathrm{a}, \mathrm{b}, \mathrm{c}, *}$ \\ ${ }^{a}$ CAS Key Laboratory of Coastal Environmental Processes and Ecological Remediation, Yantai Institute of Coastal Zone Research (YIC), Chinese Academy of Sciences \\ (CAS), Shandong Key Laboratory of Coastal Environmental Processes, YICCAS, Yantai, Shandong 264003, PR China \\ ${ }^{\mathrm{b}}$ Laboratory for Marine Biology and Biotechnology, Pilot National Laboratory for Marine Science and Technology, Qingdao, Shandong 266237, PR China \\ ${ }^{\mathrm{c}}$ Center for Ocean Mega-Science, Chinese Academy of Sciences, Qingdao, Shandong 266071, PR China
}

\section{A R T I C L E I N F O}

\section{Keywords:}

Screen-printed potentiometric sensor

Three-dimensional porous graphene aerogel

Solid contact

All-solid-state ion-selective electrode

\begin{abstract}
A B S T R A C T
An integrated all-solid-state screen-printed ion-selective potentiometric sensor for the determination of $\mathrm{Cu}^{2+}$ is proposed. The whole-cell potentiometric sensor incorporates a polymeric membrane copper ion-selective electrode $\left(\mathrm{Cu}^{2+}\right.$-ISE) as an indicator electrode and an $\mathrm{Ag} / \mathrm{AgCl}$ reference electrode with a polyvinyl butyral reference membrane. The three-dimensional (3D) self-assembled porous graphene aerogel (PGA) can be conveniently and rapidly electrodeposited on the electrode substrate surface. Due to its interior 3D interconnected porous structures, the PGA has a large electrical double layer capacitance and fast charge transfer capability, which make it promising to be used as solid contact for the fabrication of the $\mathrm{Cu}^{2+}$-ISE. The developed potentiometric sensor shows a good Nernstian response over a range from $10^{-6}$ to $10^{-3} \mathrm{M}$ with a detection limit of $10^{-6.5} \mathrm{M}$. In addition, the sensor exhibits a stable potential response with the absence of an undesirable water layer at the ionselective membrane/PGA interface.
\end{abstract}

\section{Introduction}

Heavy metal ions are not biodegradable and become a huge threat to human health and ecosystems even at low concentrations, due to negative effects of bioaccumulation and biomagnification [1]. Consequently, the precise determination of heavy metal ions is vitally important. Instruments with high precision and sensitivity, such as X-ray fluorescence spectrometer (XFS), atomic fluorescence spectrometer (AFS), atomic absorption spectrometer (AAS), and inductively coupled plasma mass spectrometer (ICP-MS) are commonly applied to detect heavy metal ions [1]. However, measurements based on these instruments need the collection and transportation of samples to central laboratories, and are time-consuming and unable to meet on-site monitoring requirements [2]. Thus, it is highly required to develop a rapid, simple and portable detection device for heavy metal ions.

The screen-printed technology is a well-developed method to fabricate cost-effective, miniaturized and portable monitoring devices for on-site analysis [3-5]. All-solid-state ion-selective electrodes (ASS-ISEs) have been widely used for detection of a variety of ions on account of their high reliability, good durability and simple operation [6-10]. In recent years, screen-printed potentiometric sensors, with the construction of ASS-ISEs on screen-printed conductive substrates, have received much attention. Various materials with excellent electrochemical properties, such as carbon nanotubes, chitosan/prussian blue nanocomposite and gold-thiol film have been used as solid contact to fabricate screen-printed potentiometric sensors [11-13]. However, these materials are usually coated on the screen-printed substrate surface through a drop-cast method, which is inappropriate for mass production of the screen-printed potentiometric sensors with reliable reproducibility.

Electrodeposition, a well-known controllable technique, has been applied as a promising method for production of uniform solid contact films in ASS-ISEs [14-16]. Conducting polymers (CPs) with unique electrical conductivities and electroactivities can be electrodeposited on electrode surfaces through the electropolymerization of monomers [14]. Unfortunately, the presence of potentially undesired side reactions and the sensitivities to $\mathrm{O}_{2}, \mathrm{CO}_{2}$ and light lead to the deterioration of potentiometric performances of CPs-based ASS-ISEs. A two-dimensional (2D) reduced graphene oxide ( $\mathrm{rGO}$ ) film formed by the electrochemical reduction of GO has been used to fabricate an ASS-ISE, which possesses excellent resistances to $\mathrm{O}_{2}, \mathrm{CO}_{2}$, light and redox interferences [16]. Although the developed ASS-ISE exhibits good potentiometric

\footnotetext{
* Corresponding author at: CAS Key Laboratory of Coastal Environmental Processes and Ecological Remediation, Yantai Institute of Coastal Zone Research (YIC), Chinese Academy of Sciences (CAS), Shandong Key Laboratory of Coastal Environmental Processes, YICCAS, Yantai, Shandong 264003, PR China.

E-mail address: wqin@yic.ac.cn (W. Qin).
} 
performances, the $2 \mathrm{D}$ rGO sheets could aggregate to form compact layered structures due to their strong van der Waals and $\pi-\pi$ stacking interactions, which may dramatically decrease the specific surface area as well as double layer capacitance of rGO [17].

Recently, the three-dimensional (3D) porous graphene aerogels (PGAs) with 3D interconnected porous structures have garnered tremendous research interest. The 3D PGAs retain the intrinsic properties of $2 \mathrm{D}$ graphene sheets and improve the practical performances of graphene in energy storage and conversion, catalysts and sensors [18,19]. A variety of methods, including template-assist, self-assembly, electrochemical reduction, thermal/electrochemical expansion, etc., have been used to prepare 3D PGAs [20]. Particularly, the electrochemical reduction method provides a simple and efficient strategy for the direct synthesis of 3D PGAs on electrode surfaces through inducing 2D graphene sheets to cross-link with each other $[17,21]$ and thus promotes the application of 3D PGAs as ideal solid contact for the construction of screen-printed ASS-ISEs.

Herein, a simple, reliable and whole-cell all-solid-state screenprinted ion-selective potentiometric sensor is proposed. The potentiometric sensor includes a polymeric membrane copper ion-selective electrode $\left(\mathrm{Cu}^{2+}\right.$-ISE) as an indicator electrode and an $\mathrm{Ag} / \mathrm{AgCl}$ reference electrode with a polyvinyl butyral (PVB) reference membrane. Self-assembled 3D PGAs used as solid contact were electrodeposited on the working electrode substrate surface through one-step electrochemical reduction of GO. The electrochemical characteristics of 3D PGA were evaluated by cyclic voltammetry (CV) and electrochemical impedance spectroscopy (EIS), and then the potentiometric performance of the fabricated screen-printed sensor was investigated.

\section{Experimental}

\subsection{Reagents}

Poly(vinyl chloride) (PVC), o-xylylene bis(diisobuty1dithiocarbamate) (copper ionophore II, $o$-XBDiBDTC), 2-nitrophenyl octyl ether ( $o$ NPOE), and sodium tetrakis[3,5-bis(trifluoro-methyl)phenyl]borate (NaTFPB) were obtained from Sigma-Aldrich. Graphite powders and all other reagents with analytical grade were purchased from Sinopharm Chemical Reagent Co., Ltd. Deionized water (18.2 M $\Omega$ cm specific resistance) from a Pall Cascada system was used to prepare all solutions. The screen-printed electrodes (SPEs) were purchased from Shenzhen Xinci Technology co. LTD. Gold ink and $\mathrm{Ag} / \mathrm{AgCl}$ ink were printed on a ceramic substrate, acting as a working electrode and a reference electrode, respectively. Dimensions of the SPE were $4.0 \mathrm{~cm} \times 1.8 \mathrm{~cm}$ (Length $\times$ Width). A photograph of the SPE is shown in Fig. S1.

\subsection{Fabrication of the all-solid-state screen-printed potentiometric sensors}

The synthesis of GO from graphite powders was performed through the modified Hummers method [22]. The 3D PGA modified SPE (SPE/ PGA) was prepared as described before $[21,23]$. The electrochemical reduction of a $3 \mathrm{mg} / \mathrm{mL} \mathrm{GO}$ aqueous dispersion containing $0.1 \mathrm{M} \mathrm{LiClO}_{4}$ at the potential of $-1.2 \mathrm{~V}$ was carried out for $10-30 \mathrm{~s}$. Then, the obtained porous graphene hydrogel (PGH) modified SPE (SPE/PGH) was further reduced in $1 \mathrm{M} \mathrm{LiClO}_{4}$ to increase the conductivity of $\mathrm{PGH}$, followed by immersion of the SPE/PGH in deionized water to remove $\mathrm{LiClO}_{4}$. Finally, the SPE/PGH was freeze-dried to obtain the SPE/PGA with 3D interconnected porous structures (Fig. S1).

$250 \mathrm{mg}$ of the sensing membrane components (1.00 wt\% copper ionophore, $0.86 \mathrm{wt} \%$ NaTFPB, $32.71 \mathrm{wt} \% \mathrm{PVC}$, and $65.43 \mathrm{wt} \% \mathrm{o}-$ NPOE) was dissolved in $2 \mathrm{ml}$ of tetrahydrofuran (THF) to prepare a membrane cocktail solution. The reference membrane cocktail was made by dissolving $50 \mathrm{mg}$ of $\mathrm{NaCl}$ and $78.1 \mathrm{mg}$ of PVB in $1 \mathrm{ml}$ of methanol [24]. The integrated screen-printed potentiometric sensor, including an indicator electrode (SPE/PGA/ $\mathrm{Cu}^{2+}$-ISE) and a solid-state $\mathrm{Ag} / \mathrm{AgCl}$ reference electrode, was obtained by drop casting $15 \mu \mathrm{l}$ of the sensing membrane solution and that of the PVB reference membrane solution on the SPE/PGA surface and the $\mathrm{Ag} / \mathrm{AgCl}$ ink electrode surface, respectively. The preparation of a coated-wire indicator electrode (SPE/ $\mathrm{Cu}^{2+}$-ISE) was the same as that of the SPE/PGA/Cu ${ }^{2+}$-ISE except for omission of the PGA modification. After drying overnight, the SPE/ $\mathrm{PGA} / \mathrm{Cu}^{2+}$-ISE and SPE $/ \mathrm{Cu}^{2+}$-ISE were conditioned in $10^{-3} \mathrm{M} \mathrm{Cu}(\mathrm{Cl})_{2}$ for $12 \mathrm{~h}$ before measurements.

\subsection{Apparatus and measurements}

The field emission scanning electron microscope (SEM) images were acquired through an S-4800 instrument (Hitachi, Ltd., Japan). Cyclic voltammetry (CV), electrochemical impedance spectroscopy (EIS) and chronopotentiometry measurements were performed by using an electrochemical workstation (CHI 660C, Shanghai Chenhua, China) with a conventional three-electrode system, which is comprised of a working electrode (SPE/PGA or SPE/PGA/Cu ${ }^{2+}$-ISE), a reference electrode (Ag/ $\mathrm{AgCl}, 3 \mathrm{M} \mathrm{KCl}$ ) and an auxiliary electrode (platinum wire).

Potentiometric measurements were carried out in $\mathrm{Cu}(\mathrm{Cl})_{2}$ solutions at concentrations in the range of $10^{-8}-10^{-3} \mathrm{M}$, using a digital ion analyzer (PXSJ-216, Shanghai Leici Instruments, China). The liquid junction potentials and the activity coefficients were corrected according to the Henderson and Debye-Hückel equations, respectively.

\section{Results and discussion}

\subsection{Characterization of the $3 D$ self-assembled PGA}

The morphologies of the 3D self-assembled PGAs are shown in Fig. 1. Due to the presence of oxygenated functionalities and electrostatic repulsion effect, uniform and random dispersion of GO sheets in water was achieved. During the electrochemical reduction of GO, the significant decrease of oxygenated functionalities and the largely restoration of $\pi$-conjugation occurred. The hydrophobic effect together with $\pi-\pi$ stacking interaction promote separated rGO sheets to partially overlap and interlock in 3D space, which could prevent restacking of rGO sheets and generate enough physical cross-link sites for the formation of interconnected porous networks [17]. It can be seen that the amount of the self-assembled PGAs is increased with the electrodeposition time. The 3D PGAs formed with the electrodeposition time of $10 \mathrm{~s}$ and $20 \mathrm{~s}$ are unable to completely cover the surface of the electrode (Fig. 1a and 1c), while well-bonded 3D porous structures with a nearly vertical arrangement of $\mathrm{rGO}$ sheets are obtained with the electrodeposition time of $30 \mathrm{~s}$ (Fig. 1e and 1f). Moreover, the photograph of the SPE/PGA is shown in Fig. S1. A dense PGA layer with the electrodeposition time of $30 \mathrm{~s}$ is formed on the SPE substrate.

As is well known, the formation of an undesirable water layer between the solid contact and ISM can be effectively prevented by using the solid contact with a high hydrophobicity, thus a contact angle of the PGA was measured. As shown in the insert of Fig. 1f, the PGA has a high hydrophobicity with a contact angle of $110 \pm 3^{\circ}(\mathrm{n}=3)$, suggesting that the PGA is a promising solid contact for ASS-ISEs.

\subsection{Electrochemical characterization of the SPE/PGA}

Electrochemical measurements of the SPE/PGA were implemented by CV and EIS, respectively. As shown in Fig. 2a, the SPE/PGA shows a much larger capacitance in comparison with the SPE/rGO and bare SPE, which is due to the $3 \mathrm{D}$ interconnected porous structures of PGA [25]. The impedance plot of the SPE/PGA is dominated by an approximate $90^{\circ}$ capacitive line with the absence of a high-frequency semicircle, while the capacitive line of SPE/rGO is apparently deviated in comparison with that of the SPE/PGA (Fig. 2b). These results indicate the presence of fast charge transfers at the SPE/PGA and PGA/solution interfaces along with good conductivity of PGA. 

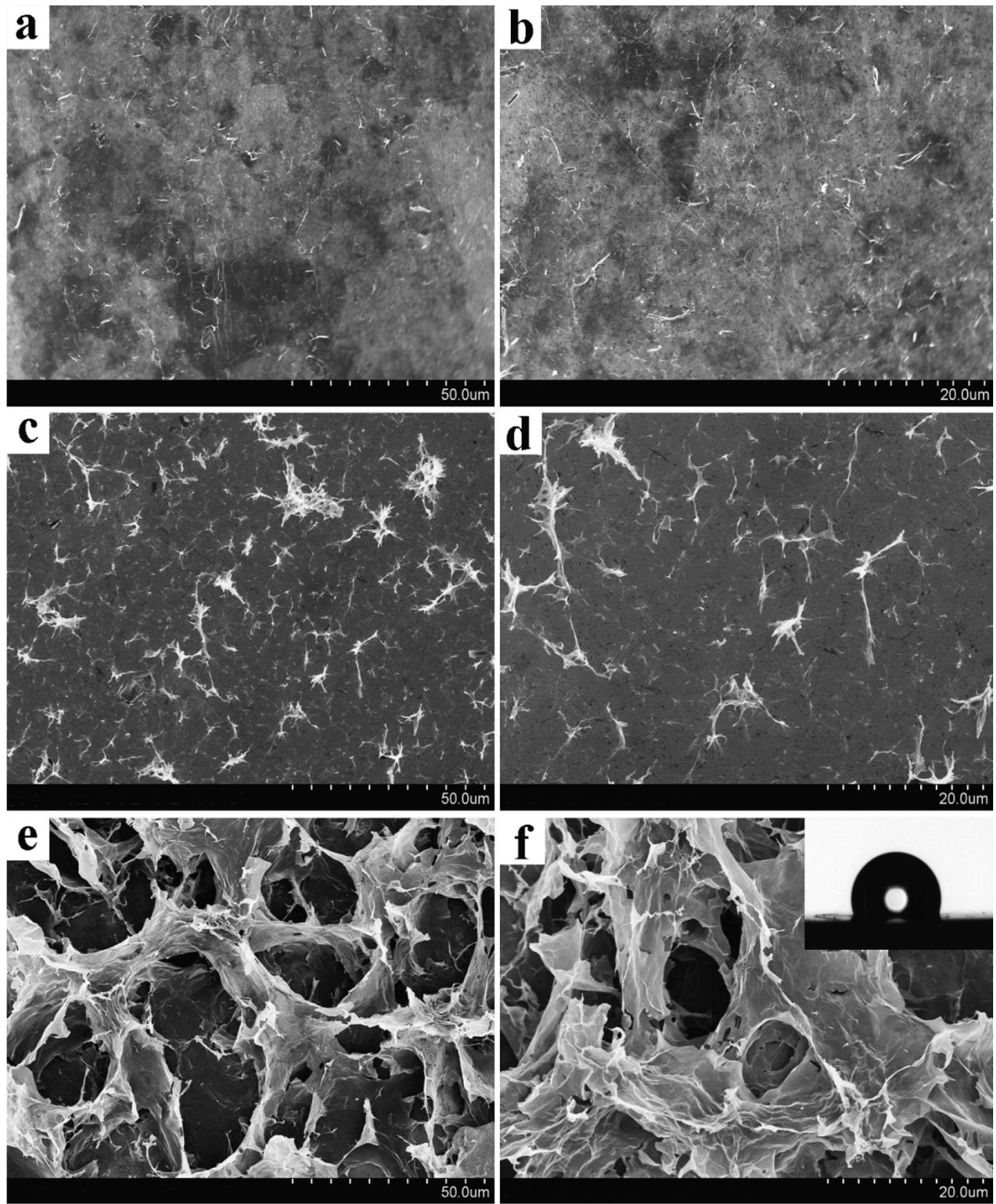

Fig. 1. SEM images of PGA with (a, c and e) low and (b, d and f) high magnifications. The electrodeposited times were 10, 20 and $30 \mathrm{~s}$, respectively. The inset in Fig. 1f shows the contact angle of PGA with the electrodeposited time of $30 \mathrm{~s}$.

\subsection{Potentiometric performance of the integrated screen-printed sensor}

The dynamic potentiometric response traces of the integrated screen-printed sensor tested in $\mathrm{Cu}(\mathrm{Cl})_{2}$ solutions at concentrations ranging from $10^{-3}$ to $10^{-8} \mathrm{M}$ are illustrated in Fig. 3a. The corresponding calibration curve is shown in Fig. 3b. As can be seen, the proposed screen-printed potentiometric sensor shows a Nernstian response with a slope of $28.7 \pm 1.5 \mathrm{mV} /$ decade $\left(n=4, R^{2}=0.9920\right)$ in the range of $10^{-6}-10^{-3} \mathrm{M}$. The detection limit is $10^{-6.5} \mathrm{M}$. Selectivity coefficients of the SPE/PGA/Cu ${ }^{2+}$-ISE were examined by the separated solution method ( $n=3$ ) [26]. As shown in Fig. 4, selectivity coefficients obtained for the SPE/PGA/Cu ${ }^{2+}$-ISE agree well with those from the reported literature [27], indicating that the selectivity of ISEs is related to the sensing membrane itself instead of the solid contact.

\subsection{Potential stability}

The chronopotentiometry measurements were performed in $0.1 \mathrm{M}$ $\mathrm{Cu}(\mathrm{Cl})_{2}$ to evaluate the short-term potential stability of the SPE/PGA/ $\mathrm{Cu}^{2+}$-ISE. The typical chronopotentiograms for the SPE/PGA/Cu${ }^{2+}$-ISE and SPE $/ \mathrm{Cu}^{2+}$-ISE are exhibited in Fig. 5. The potential drift $(\Delta E / \Delta t)$ is calculated by the equation $\Delta E / \Delta t=I / C$ [28], associating with the lowfrequency capacitance $(C)$ and the applied constant current $(I)$. The potential drift of the SPE/PGA/ $\mathrm{Cu}^{2+}$-ISE is $1.2 \pm 0.2 \mu \mathrm{Vs}^{-1}(\mathrm{n}=3)$, which is much less than the value $\left(246 \mu \mathrm{Vs}^{-1}\right)$ obtained with the SPE/ $\mathrm{Cu}^{2+}$-ISE. The low-frequency capacitance for the SPE/PGA/ $\mathrm{Cu}^{2+}$-ISE is $833 \mu \mathrm{F}$, which is larger than those of the SPE/ $\mathrm{Cu}^{2+}$-ISE $(2.3 \mu \mathrm{F})$.

The long-term stability and lifetime of the SPE/PGA/ $\mathrm{Cu}^{2+}$-ISE were also investigated due to that the adhesion between ISM and substrate might decrease in a long time, resulting in the deterioration of potential 

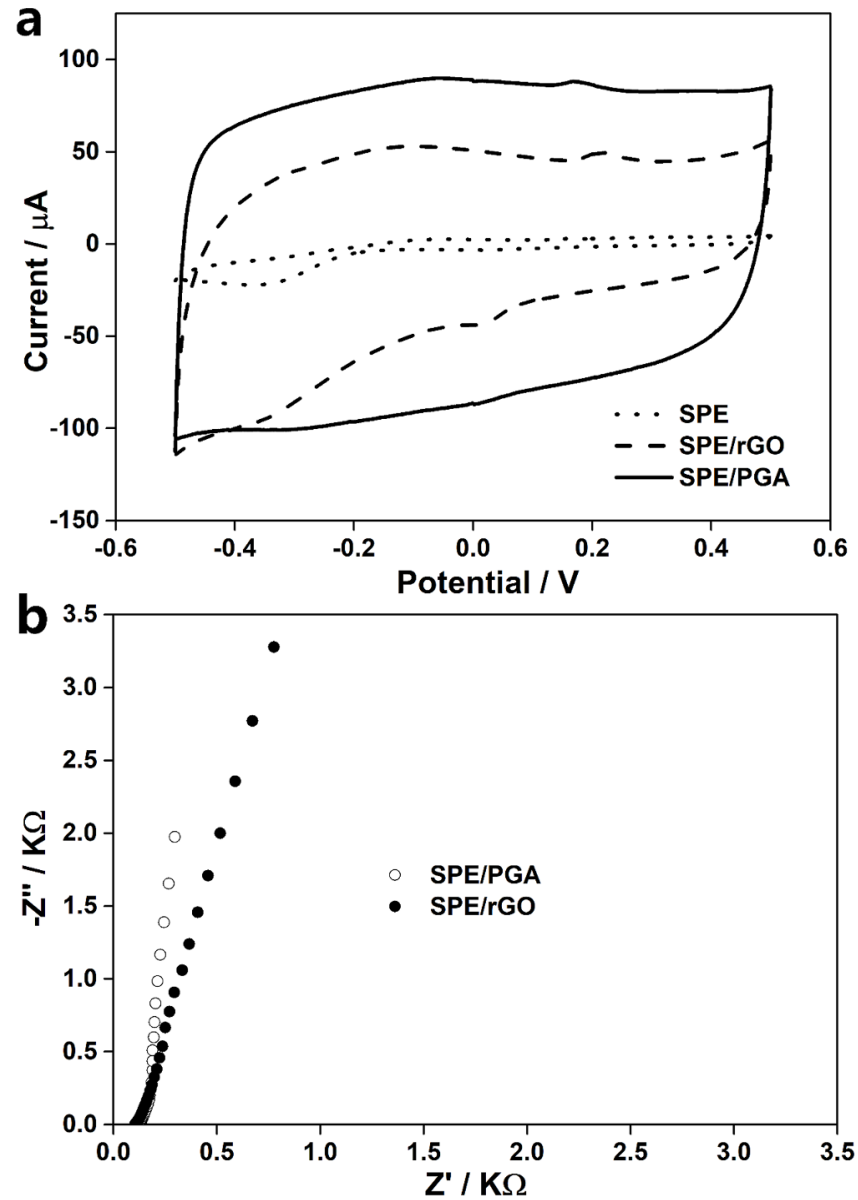

Fig. 2. (a) Cyclic voltammograms of the SPE/PGA, SPE/rGO and SPE electrodes in $0.1 \mathrm{M} \mathrm{KCl}$ at a scan rate of $100 \mathrm{mV} \mathrm{s}^{-1}$. (b) Impedance spectra of the SPE/ PGA and SPE/rGO electrodes in $0.1 \mathrm{M} \mathrm{KCl}$ at the open-circuit potential. Initial potential, $0.2 \mathrm{~V}$; excitation amplitude, $10 \mathrm{mV}$; frequency range, $0.3 \mathrm{~Hz}$ to $10 \mathrm{kHz}$.

responses [29]. The long-term stability test of SPE/PGA/ $\mathrm{Cu}^{2+}$-ISEs was continuously performed for $24 \mathrm{~h}$ in $0.1 \mathrm{M} \mathrm{Cu}(\mathrm{Cl})_{2}$. The potential drift of $8.5 \pm 0.5 \mu \mathrm{Vh}^{-1}(\mathrm{n}=3)$ for the SPE/PGA/Cu${ }^{2+}$-ISE is shown in Fig. $\mathrm{S} 2$, indicating an excellent long-term stability of the SPE/PGA/ $\mathrm{Cu}^{2+}$. ISE. Potentiometric properties of ASS-ISEs with various nanomaterials used as solid contact are given in Table S1. The short-term and longterm potential stabilities of the SPE/PGA/ $\mathrm{Cu}^{2+}$-ISE are greatly enhanced in the use of PGA as solid contact. Superior potential stabilities of the SPE/PGA/ $\mathrm{Cu}^{2+}$-ISE are attributed to the high double layer capacitance of the PGA. The lifetime behaviors of the SPE/PGA/ $\mathrm{Cu}^{2+}$-ISE were tested by recording potential responses of the same electrode during a period of 15 days $(n=3)$. As shown in Fig. S3, the detection limit and the slope of the SPE/PGA/Cu ${ }^{2+}$-ISE are barely changed after continuous measurements for 15 days.

\subsection{Impedance measurements}

Fig. 6 shows the impedance spectra of the SPE/PGA/ $\mathrm{Cu}^{2+}$-ISE and $\mathrm{SPE} / \mathrm{Cu}^{2+}$-ISE. It is notable that the exhibited high-frequency semicircle represents the bulk resistance including sensing membrane resistance and the contact resistance at the sensing membrane/PGA or SPE interface. The high-frequency resistance values of the SPE/PGA/ $\mathrm{Cu}^{2+}$-ISE and SPE $/ \mathrm{Cu}^{2+}$-ISE are 39 and $44 \mathrm{k} \Omega$, respectively, indicating that the SPE/PGA/ $\mathrm{Cu}^{2+}$-ISE has a low charge transfer resistance at the sensing membrane/PGA interface [30]. In addition, in the low-frequency part, the semicircle of the SPE/PGA/ $\mathrm{Cu}^{2+}$-ISE is relatively
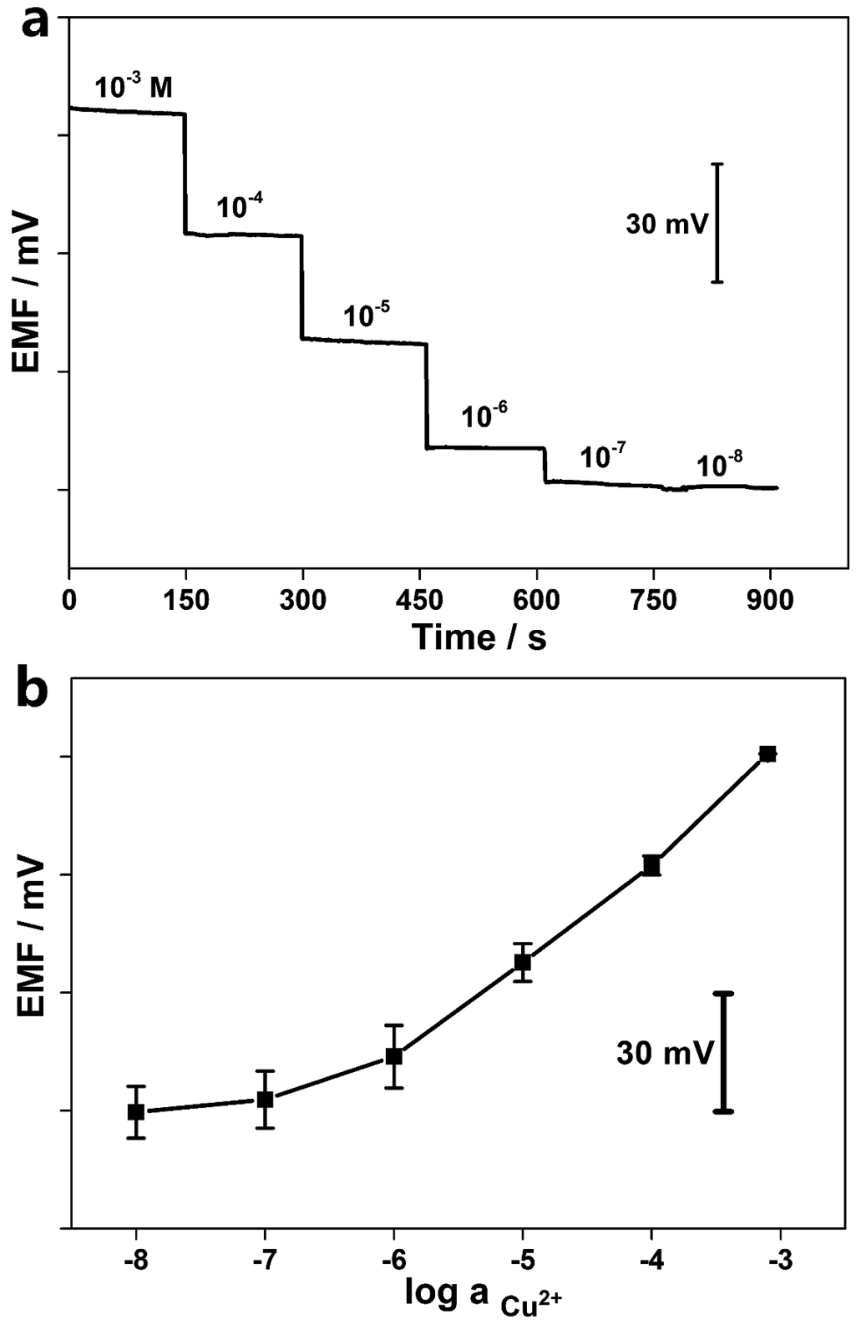

Fig. 3. (a) Time-dependent potential responses and (b) calibration curve for the integrated screen-printed sensor in the range of $10^{-3}-10^{-8} \mathrm{M}$.

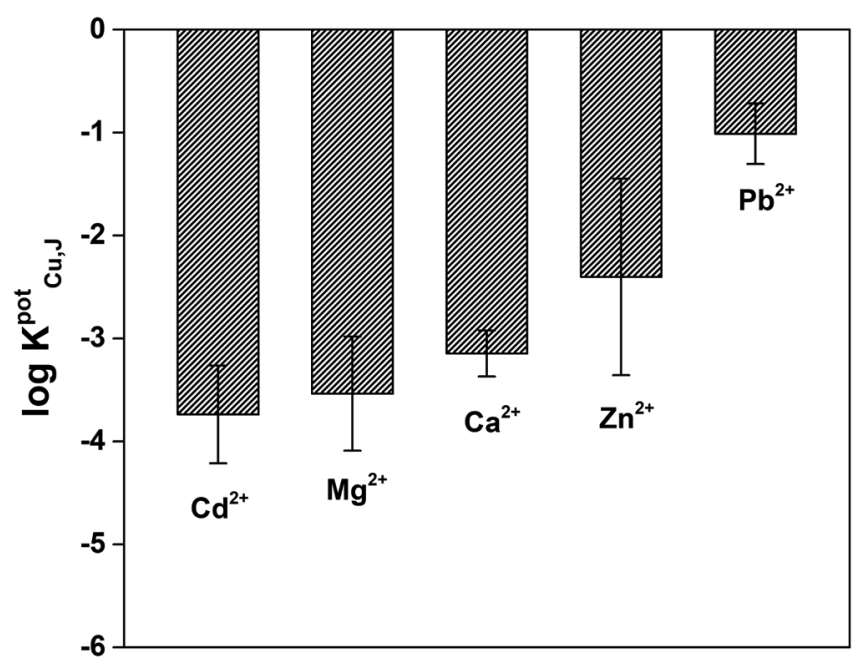

Fig. 4. Selectivity coefficients $\left(\log K_{C u, J}^{\text {pot }}\right)$ obtained with the separate solution method for the SPE/PGA/Cu ${ }^{2+}$-ISE.

smaller than that of the SPE/ $\mathrm{Cu}^{2+}$-ISE. These results demonstrate the existence of a low charge transfer resistance as well as a high double layer capacitance at the sensing membrane/PGA interface. 


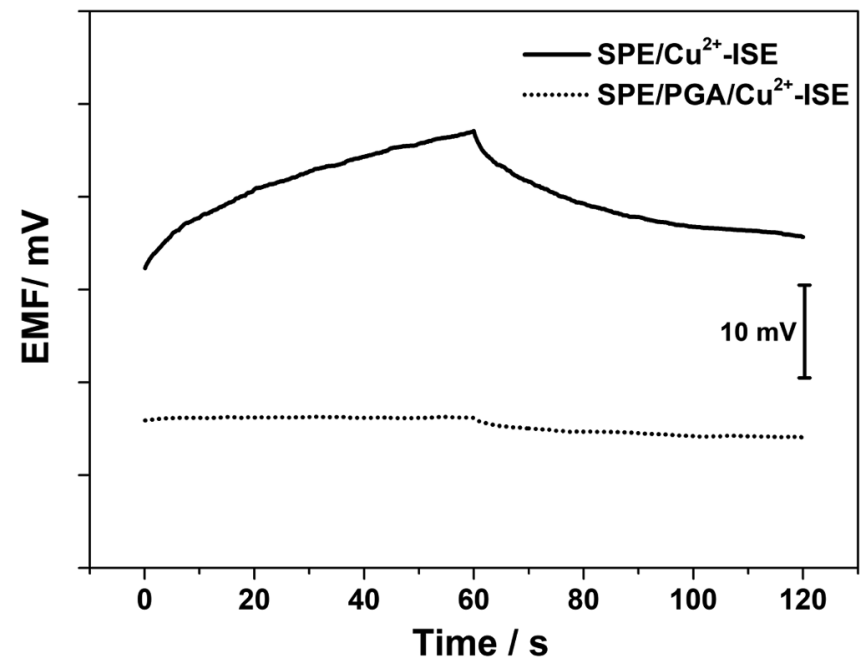

Fig. 5. Chronopotentiograms of the SPE/PGA/Cu ${ }^{2+}$-ISE and SPE/Cu ${ }^{2+}$-ISE at the applied currents of $\pm 1 \mathrm{nA}$ for $60 \mathrm{~s}$.
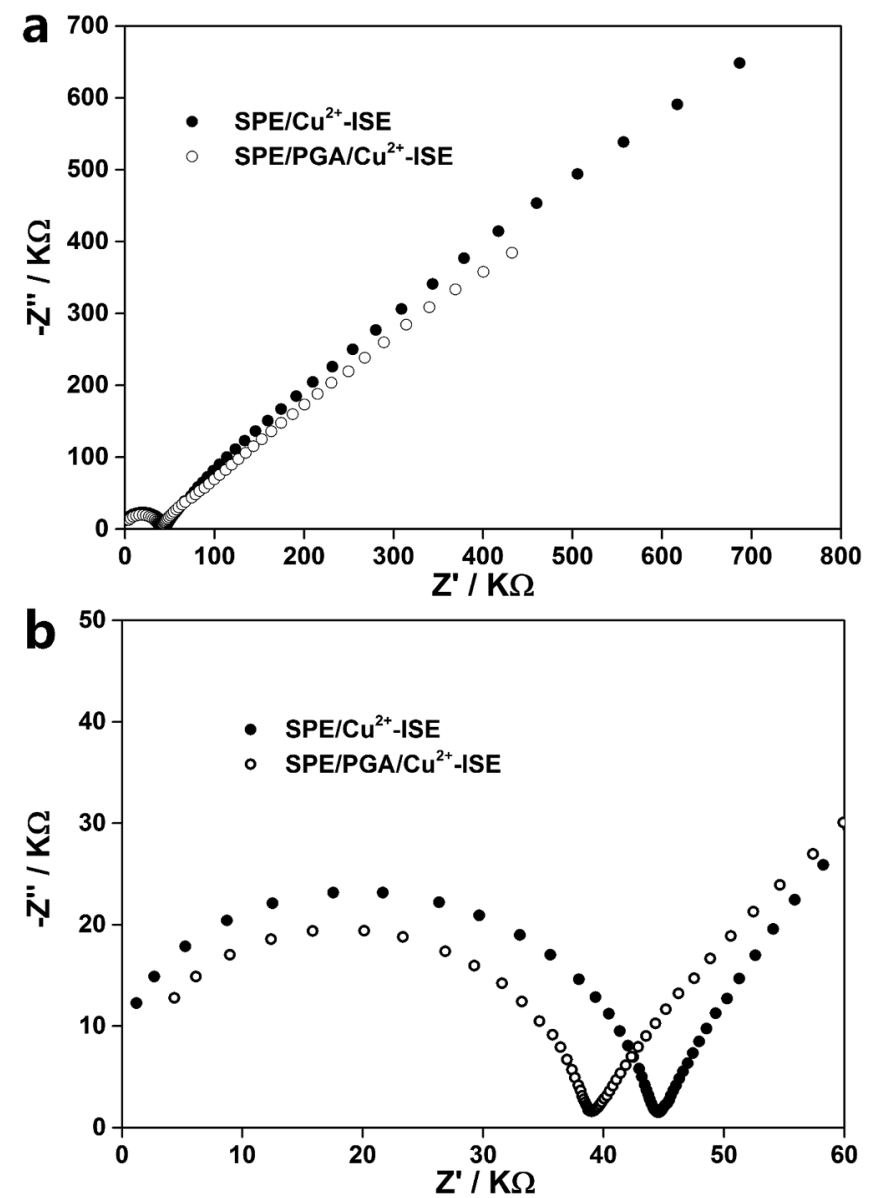

Fig. 6. (a) Impedance plots of the $\mathrm{SPE} / \mathrm{PGA} / \mathrm{Cu}^{2+}$-ISE and $\mathrm{SPE} / \mathrm{Cu}^{2+}-\mathrm{ISE}$. Initial potential, $0.2 \mathrm{~V}$; excitation amplitude, $100 \mathrm{mV}$; frequency range, 0.01-100 kHz. (b) The magnification of the impedance spectra.

\subsection{Effects of Light, $\mathrm{O}_{2}$, and $\mathrm{CO}_{2}$}

The interferences of $\mathrm{CO}_{2}, \mathrm{O}_{2}$, and light on the potential stability of the SPE/PGA/ $\mathrm{Cu}^{2+}$-ISE were studied. Effects of $\mathrm{CO}_{2}$ and $\mathrm{O}_{2}$ on the SPE/ $\mathrm{PGA} / \mathrm{Cu}^{2+}$-ISE were tested by recording the potential responses of the proposed electrode in $0.1 \mathrm{M} \mathrm{Cu}(\mathrm{Cl})_{2}$ under the condition of bubbling $\mathrm{CO}_{2}$ and $\mathrm{N}_{2}$, or $\mathrm{O}_{2}$ and $\mathrm{N}_{2}$ for $30 \mathrm{~min}$. The interference of light is

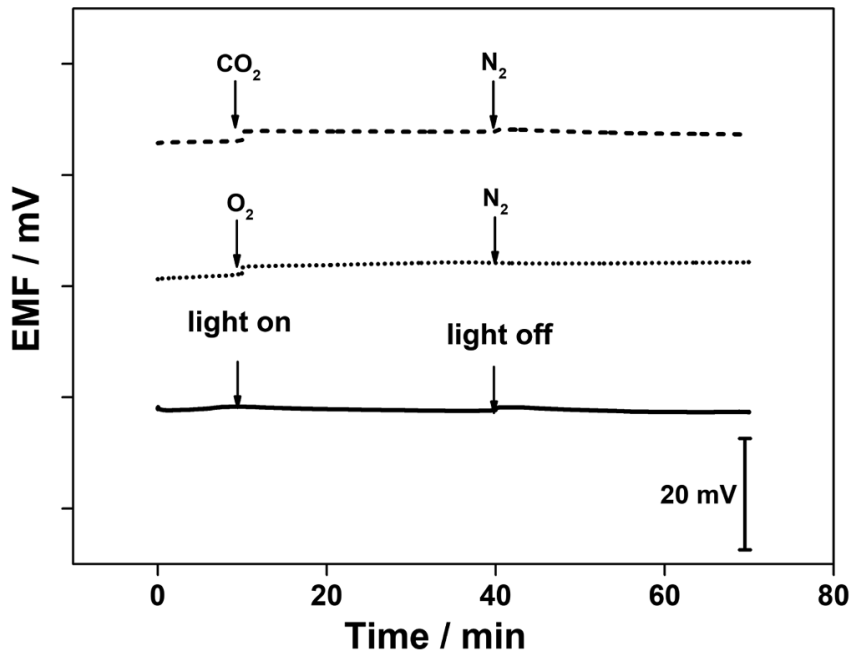

Fig. 7. Effects of $\mathrm{CO}_{2}, \mathrm{O}_{2}$ and light on the potential stability of the SPE/PGA/ $\mathrm{Cu}^{2+}$-ISE.

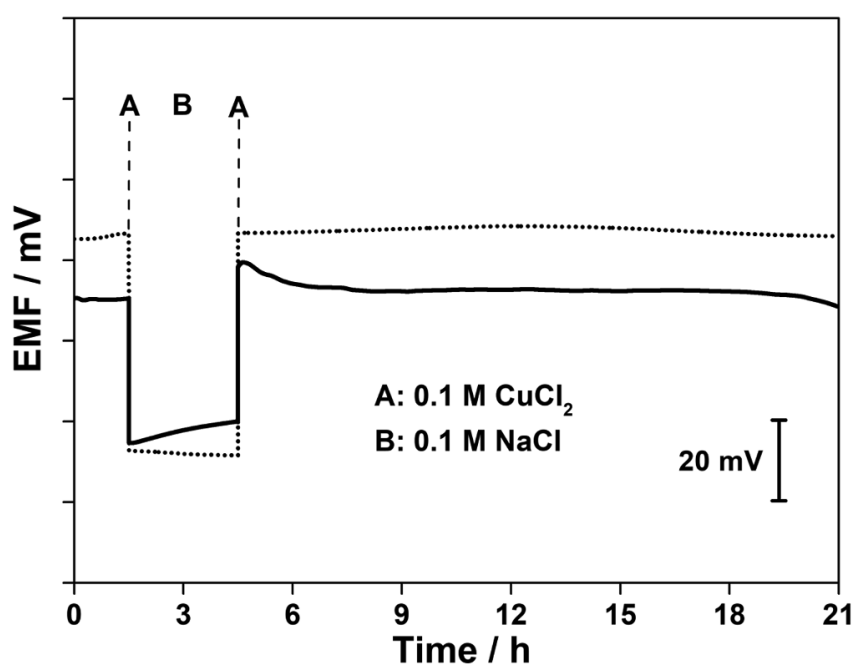

Fig. 8. Water layer tests for the SPE/PGA/Cu ${ }^{2+}$-ISE (dotted line) and SPE/ $\mathrm{Cu}^{2+}$-ISE (solid line).

measured by immersing the PGA-based electrode in $0.1 \mathrm{M} \mathrm{Cu}(\mathrm{Cl})_{2}$ with the ambient light on/off. As can be seen in Fig. 7, potential drifts can hardly be found during the presence of $\mathrm{CO}_{2}, \mathrm{O}_{2}$ and light, indicating that the SPE/PGA/ $\mathrm{Cu}^{2+}$-ISE is robust to the interferences from $\mathrm{CO}_{2}, \mathrm{O}_{2}$ and light.

\subsection{Water layer test}

To investigate the influence of a water layer between the sensing membrane and PGA on the potential stability of the SPE/PGA/Cu ${ }^{2+}$. ISE, the water layer test was carried out [25]. The SPE/PGA/Cu ${ }^{2+}$-ISE and SPE $/ \mathrm{Cu}^{2+}$-ISE were sequentially immersed in $0.1 \mathrm{M} \mathrm{Cu}(\mathrm{Cl})_{2}, 0.1 \mathrm{M}$ $\mathrm{NaCl}$, and $0.1 \mathrm{M} \mathrm{Cu}(\mathrm{Cl})_{2}$. In Fig. 8, compared with the SPE/ $\mathrm{Cu}^{2+}$-ISE, the SPE/PGA/ $\mathrm{Cu}^{2+}$-ISE shows a stable response during the water layer test, which demonstrates the absence of a water layer at the sensing membrane/PGA interface, probably due to the resultant hydrophobic characteristic of electrochemically reduced 3D PGA [17].

\subsection{Analytical applications}

To further investigate the feasibility of the SPE/PGA/ $\mathrm{Cu}^{2+}$-ISE for environmental analysis, $\mathrm{Cu}^{2+}$ standard solutions with different 
Table 1

Recovery tests obtained by the proposed potentiometric sensor for copper in tap water samples. ${ }^{\text {a }}$

\begin{tabular}{lclc}
\hline Sample & Added $\left(\times 10^{-6} \mathrm{M}\right)$ & Found $\left(\times 10^{-6} \mathrm{M}\right)$ & Recovery (\%) \\
\hline Tap water 1 & 1.0 & $1.02 \pm 0.14$ & 102.2 \\
Tap water 2 & 5.0 & $4.83 \pm 0.23$ & 96.6 \\
Tap water 3 & 10.0 & $9.50 \pm 0.89$ & 95.0 \\
\hline
\end{tabular}

a Average value of three determinations \pm standard deviation.

contents were spiked into the tap water samples, and then the $\mathrm{Cu}^{2+}$ in mixed samples were detected with the proposed electrode. Percent recovery values were calculated and shown in Table 1 . As can be seen in Table 1 , recoveries of the $\mathrm{Cu}^{2+}$ determination in different tap water samples were in a range of $95.0 \%-102.2 \%$. These results indicate that the developed SPE/PGA/Cu ${ }^{2+}$-ISE can be used to detect $\mathrm{Cu}^{2+}$ reliably in real samples.

\section{Conclusions}

An integrated screen-printed potentiometric sensor, which is comprised of an all-solid-state polymeric membrane $\mathrm{Cu}^{2+}$-selective electrode and an all-solid-state $\mathrm{Ag} / \mathrm{AgCl}$ reference electrode has been developed. The $\mathrm{Cu}^{2+}$-selective indicator electrode is fabricated using 3D PGA as solid contact, which can be readily formed on the gold ink substrate surface by the electrodeposition method. The PGA with 3D interconnected porous structures possesses excellent properties such as fast charge transfer, high specific surface area and large double layer capacitance. The proposed all-solid-state screen-printed potentiometric sensor exhibits a stable Nernstian response, high potential stability and small impedance. No undesirable water layer is formed at the sensing membrane/PGA interface.

\section{CRediT authorship contribution statement}

Jinghui Li: Investigation, Writing - original draft. Wei Qin: Supervision, Writing - review \& editing.

\section{Declaration of Competing Interest}

The authors declare that they have no known competing financial interests or personal relationships that could have appeared to influence the work reported in this paper.

\section{Acknowledgements}

This work was financially supported by the National Natural Science Foundation of China (21677172), the National Key Research and Development Program of China (2016YFC1400700), the Taishan Scholar Program of Shandong Province (TSPD20181215).

\section{Appendix A. Supplementary data}

Supplementary data to this article can be found online at https:// doi.org/10.1016/j.microc.2020.105453.

\section{References}

[1] L.A. Malik, A. Bashir, A. Qureashi, A.H. Pandith, Detection and removal of heavy metal ions: a review, Environ. Chem. Lett. 17 (2019) 1495-1521.

[2] A. Hayat, J.L. Marty, Disposable screen printed electrochemical sensors: tools for environmental monitoring, Sensors 14 (2014) 10432-10453.

[3] J.P. Jasmin, F. Miserque, E. Dumas, I. Vickridge, J.J. Ganem, C. Cannizzo, A. Chaussé, XPS and NRA investigations during the fabrication of gold nanostructured functionalized screen-printed sensors for the detection of metallic pollutants, Appl. Surf. Sci. 397 (2017) 159-166.

[4] X. Liu, Y. Yao, Y. Ying, J. Ping, Recent advances in nanomaterial-enabled screenprinted electrochemical sensors for heavy metal detection, TrAC, Trends Anal. Chem. 115 (2019) 187-202.

[5] T. Yin, H. Yu, J. Ding, W. Qin, An integrated screen-printed potentiometric strip for determination of $\mathrm{Ca}^{2+}$ in seawater, J. Electrochem. Soc. 166 (2019) B589-B593.

[6] Y. Liu, Y. Liu, Y. Gao, P. Wang, A general approach to one-step fabrication of singlepiece nanocomposite membrane based $\mathrm{Pb}^{2+}$-selective electrodes, Sens. Actuators, B Chem. 281 (2019) 705-712.

[7] J. Ping, Y. Wang, K. Fan, W. Tang, J. Wu, Y. Ying, High-performance flexible potentiometric sensing devices using free-standing graphene paper, J. Mater. Chem. B 1 (2013) 4781-4791.

[8] Y. Shao, Y. Ying, J. Ping, Recent advances in solid-contact ion-selective electrodes: functional materials, transduction mechanisms, and development trends, Chem. Soc. Rev. 49 (2020) 4405-4465.

[9] E. Zdrachek, E. Bakker, Potentiometric sensing, Anal. Chem. 91 (2019) 2-26.

[10] G. Zhao, R. Liang, F. Wang, J. Ding, W. Qin, An all-solid-state potentiometric microelectrode for detection of copper in coastal sediment pore water, Sens. Actuators, B Chem. 279 (2019) 369-373.

[11] T. Ghosh, H.-J. Chung, J. Rieger, All-solid-state sodium-selective electrode with a solid contact of chitosan/prussian blue nanocomposite, Sensors 17 (2017) 2536.

[12] M. Li, H. Zhou, L. Shi, D. Li, Y. Long, Ion-selective gold-thiol film on integrated screen-printed electrodes for analysis of Cu(II) ions, Analyst 139 (2014) 643-648.

[13] F.X. Rius-Ruiz, G.A. Crespo, D. Bejarano-Nosas, P. Blondeau, J. Riu, F.X. Rius, Potentiometric strip cell based on carbon nanotubes as transducer layer: toward low-cost decentralized measurements, Anal. Chem. 83 (2011) 8810-8815.

[14] J. Bobacka, Conducting polymer-based solid-state ion-selective electrodes, Electroanalysis 18 (2006) 7-18.

[15] F. Criscuolo, I. Taurino, F. Stradolini, S. Carrara, G. De Micheli, Highly-stable Li ${ }^{+}$ ion-selective electrodes based on noble metal nanostructured layers as solid-contacts, Anal. Chim. Acta 1027 (2018) 22-32.

[16] J. Ping, Y. Wang, Y. Ying, J. Wu, Application of electrochemically reduced graphene oxide on screen-printed ion-selective electrode, Anal. Chem. 84 (2012) 3473-3479.

[17] Y. Xu, G. Shi, X. Duan, Self-assembled three-dimensional graphene macrostructures: synthesis and applications in supercapacitors, Acc. Chem. Res. 48 (2015) $1666-1675$.

[18] Y. Ma, Y. Chen, Three-dimensional graphene networks: synthesis, properties and applications, Natl. Sci. Rev. 2 (2014) 40-53.

[19] X. Yao, Y. Zhao, Three-dimensional porous graphene networks and hybrids for lithium-ion batteries and supercapacitors, Chem 2 (2017) 171-200.

[20] Y. Wu, J. Zhu, L.u. Huang, A review of three-dimensional graphene-based materials: synthesis and applications to energy conversion/storage and environment, Carbon 143 (2019) 610-640.

[21] K. Sheng, Y. Sun, C. Li, W. Yuan, G. Shi, Ultrahigh-rate supercapacitors based on eletrochemically reduced graphene oxide for ac line-filtering, Sci. Rep. 2 (2012) 247.

[22] Y. Xu, K. Sheng, C. Li, G. Shi, Self-assembled graphene hydrogel via a one-step hydrothermal process, ACS Nano 4 (2010) 4324-4330.

[23] K. Chen, L. Chen, Y. Chen, H. Bai, L. Li, Three-dimensional porous graphene-based composite materials: electrochemical synthesis and application, J. Mater. Chem. 22 (2012) 20968.

[24] T. Guinovart, A.J. Bandodkar, J.R. Windmiller, F.J. Andrade, J. Wang, A potentiometric tattoo sensor for monitoring ammonium in sweat, Analyst 138 (2013) 7031-7038.

[25] J. Hu, A. Stein, P. Bühlmann, Rational design of all-solid-state ion-selective electrodes and reference electrodes, TrAC, Trends Anal. Chem. 76 (2016) 102-114.

[26] E. Bakker, E. Pretsch, P. Bühlmann, Selectivity of potentiometric ion sensors, Anal. Chem. 72 (2000) 1127-1133.

[27] S. Kamata, H. Murata, Y. Kubo, A. Bhale, Copper(II)-selective membrane electrodes based on o-xylylene bis(dithiocarbamates) as neutral carriers, Analyst 114 (1989) 1029-1031.

[28] J. Bobacka, Potential stability of all-solid-state ion-selective electrodes using conducting polymers as ion-to-electron transducers, Anal. Chem. 71 (1999) 4932-4937.

[29] E. Lindner, V.V. Cosofret, S. Ufer, R.P. Buck, R.P. Kusy, R.B. Ash, H.T. Nagle, Flexible (Kapton-based) microsensor arrays of high stability for cardiovascular applications, Faraday Trans. 89 (1993) 361-367.

[30] B. Paczosa-Bator, Ion-selective electrodes with superhydrophobic polymer/carbon nanocomposites as solid contact, Carbon 95 (2015) 879-887. 